\title{
Infected thoracoabdominal aortic aneurysm related to an implanted long-term arterial catheter for chemotherapy: a case report
}

\author{
Kiyoshi Takemoto $^{1 *}\left(\mathbb{D}\right.$, Michitaka Nakamura $^{1}$, Masaaki Sakuraya ${ }^{1,2}$, Tomonori Yamamoto ${ }^{1}$, Wataru Iwanaga ${ }^{1}$, \\ Kazuaki Atagi ${ }^{1}$, Kazuo Yamanaka ${ }^{3}$ and Takeshi Matsuyama ${ }^{1}$
}

\begin{abstract}
Background: An infected aortic aneurysm is a rare and life-threatening vascular condition with a high incidence of arterial rupture and recurrence even after treatment. One of the most common causes of an infected aortic aneurysm is catheter-related bloodstream infection. Although infection due to indwelling catheters is possible, the incidence of this is rare, especially for long-term implanted arterial catheters.
\end{abstract}

Case presentation: A 78-year-old Japanese man with a past medical history of rectal cancer with metastasis to the liver presented to our hospital as a result of low back pain. Remission had been achieved following surgery and adjuvant chemotherapy via an implanted catheter for arterial infusion. However, the original catheter that was inserted from the femoral artery to the hepatic artery via the celiac artery was still present more than 10 years after diagnosis, without being replaced, in case of a recurrence. On the day of admission, computed tomography scan of the chest and abdomen with contrast revealed an irregularly shaped aortic aneurysm at the origin of the celiac artery and a partially expanded common hepatic artery with disproportionate fat stranding along the implanted arterial catheter without extravasation. Although the initial impression was an impending rupture of the acute thoracoabdominal aortic aneurysm, a catheter-related infection was considered as a differential diagnosis. Surgery was performed, which revealed a catheter-related infected aortic aneurysm based on images along the catheter, pus cultures, and tissue pathology examination results.

Conclusions: This is an extremely rare case of an infectious aneurysm caused by prolonged implantation of an arterial catheter for chemotherapy. It should be noted that an indwelling arterial catheter not only causes bloodstream infections but can also cause an infection of a thoracoabdominal aortic aneurysm.

Keywords: Infected thoracoabdominal aneurysm, Implants, Arterial catheter

\section{Background}

An infected aortic aneurysm a rare life-threatening vascular condition which can eventually result in rupture of the arterial wall if left untreated. When infected aortic aneurysm is suspected, immediate confirmatory

\footnotetext{
*Correspondence: kiyo17868@gmail.com

1 Division of Critical Care Medicine, Nara Prefecture General Medical Center, Shichijyonishi 2-897-5, Nara City, Nara, Japan

Full list of author information is available at the end of the article
}

diagnosis and definitive treatment are essential [1]. As there are no definitive diagnostic criteria for infected aneurysms, radiographic imaging, blood and tissue cultures, and tissue pathology examinations must be evaluated. Negative blood cultures are not enough to exclude infected aneurysm because positive blood cultures can be obtained in only $50-70 \%$ of patients with an infected aneurysm [2,3]. Despite report of venous and arterial infections aneurysms due to catheters [4], we could not find published works reporting infected aneurysms due 
to long-term implanted catheters. This was an extremely rare case of an infectious aneurysm caused by prolonged implantation of an arterial catheter. This case report suggests that an indwelling arterial catheter not only causes bloodstream infections but can also cause an infection of a thoracoabdominal aortic aneurysm.

\section{Case presentation}

A 78-year-old Japanese male presented to the emergency department of our hospital with low back pain on exertion for 1 week. The pain was described as dull and gradually worsens. Although the location was near the thoracolumbar spine, he denied radiation of the pain to any parts of the body. Severity of pain using a numerical rating scale was $10 / 10$ at the day of admission. The character and intensity of the pain were not affected by changes in physical movement or by rest. He denied any other symptoms such as fever, nausea, dysuria, hematuria, abdominal pain, and leg numbness during his clinical course.

He had a past medical history of rectal cancer with liver metastasis and had undergone surgery and chemotherapy. At the time of diagnosis, rectal cancer was stage IV (TNM classification of malignant tumors; T3N2M1), grade 3, and was revealed to be adenocarcinoma during histopathology. Liver metastasis affected segments 3 and 6 . He underwent low anterior resection of the rectum and resection of the affected liver segments. He then underwent chemotherapy using fluorouracil that was arterially infused through a catheter inserted into the femoral artery and implanted into the hepatic artery through the celiac artery. He initially had good response to treatment but 2 years after diagnosis, he had a recurrence of liver metastasis. He underwent partial resection of segment 6 of the liver and was followed by chemotherapy using FOLFOX6 + bevacizumab protocol instead of arterial infusion. After finishing chemotherapy, he achieved complete remission 11 years after initial diagnosis. As a result of the possibility of another recurrence, the catheter remained in place without being replaced. His other past medical history was hypertension and he remained on amlodipine $5 \mathrm{mg}$ daily and imidapril $5 \mathrm{mg}$ daily. Social history revealed that he had smoked approximately 10 cigarettes a day for 50 years and drank alcohol occasionally. Family and environmental history was unremarkable. His employment history was an office worker, but he retired at the age of 60 and has not worked since then.

On the day of admission, his blood pressure was $171 / 75 \mathrm{mmHg}$, heart rate was $67 \mathrm{bpm}, \mathrm{SpO}_{2} 97 \%$ at ambient room air, and body temperature was $36.6{ }^{\circ} \mathrm{C}$. $\mathrm{He}$ denied abdominal pain, and pain or numbness in the lower extremities. General appearance was not in acute distress. There was no conjunctiva pallor or icterus. Respiratory sounds were clear to auscultation bilaterally and there were no wheezes or crackles. Cardiovascular examination revealed normal S1 and S2. There was no S3, S4, or murmurs. Abdominal examination revealed a flat and soft abdomen with audible bowel sounds. There was no bruit. There was no abdominal tenderness or hepatosplenomegaly. There was no spinal tenderness or costovertebral angle tenderness on percussion. There was no edema of his lower extremities. There was no joint swelling bilaterally at the wrists, ankles, and knees. General physical examinations revealed no abnormalities. His neurologic examination 2 to 12 were intact. There were no abnormalities with sensation and strength throughout with normal reflexes. Although laboratory analysis revealed normal results for complete blood count, electrolyte level, creatinine level, liver function, and coagulation test, levels of beta-D-glucan were slightly elevated at $24 \mathrm{pg} / \mathrm{mL}$ (reference value, $<20 \mathrm{pg} / \mathrm{mL}$ ) (Table 1). Urinalysis was negative for proteinuria, pyuria, and hematuria (Table 1). Blood culture of aerobic and anaerobic bacteria including fungi and urine culture were all negative (Table 1). Transthoracic echocardiography revealed no valve vegetation, no valve regurgitation, no stenosis, and a normal ejection fraction. Computed tomography (CT) of the chest and abdomen revealed an irregularly shaped aortic aneurysm measuring $45 \times 33 \mathrm{~mm}$ at the origin of the celiac artery and a partially expanded common hepatic artery with disproportionate fat stranding; no extravasation was observed using contrast enhancement (Fig. 1). There was a high possibility that the aortic aneurysm was infected because it was at the site of the catheter that was inserted for the femoral artery via the common hepatic artery. The patient was diagnosed with impending rupture of acute thoracoabdominal aortic aneurysm and was admitted to the intensive care unit of our hospital. Graft replacement was performed for the thoracoabdominal aortic aneurysm, and the implanted catheter was removed during surgery and tested for culture. Pus was discharged from the aortic aneurysm wall incision and collected with swab for culture. The cultures of both the removed catheter and the pus of the aneurysm revealed Escherichia coli, Serratia marcescens, Eikenella corrodens, Streptococcus anginosus, $\alpha$-Streptococcus, and Candida glabrata. The reported antimicrobial sensitivities of these organisms are shown in Table 2. Antimicrobial susceptibilities were determined by the disk diffusion method, and the results were interpreted according to the Clinical and Laboratory Standards Institute (CLSI) guidelines. Results of pathology examination of the wall tissue of the aneurysm were compatible with those of the infected aneurysm cultures because the former showed infiltration of neutrophils mainly in the small blood vessels around the 
Table 1 Results of laboratory findings

\begin{tabular}{|c|c|c|c|c|c|c|c|c|}
\hline \multicolumn{3}{|l|}{ Complete blood count } & \multicolumn{3}{|l|}{ Biochemistry test } & \multicolumn{3}{|l|}{ Urinalysis } \\
\hline White blood cell & 7.6 & $10^{3} / \mu \mathrm{L}$ & Total protein & 7.8 & $\mathrm{~g} / \mathrm{dL}$ & Dipstick & & \\
\hline Neutrophils & 69.1 & $\%$ & Albumin & 3.6 & $\mathrm{~g} / \mathrm{dL}$ & Color & Yellow & \\
\hline Lymphocytes & 22.0 & $\%$ & Aspartate aminotransferase & 16.0 & $\mathrm{IU} / \mathrm{L}$ & Specific gravity & 1.024 & \\
\hline Eosinophils & 0.8 & $\%$ & Alanine aminotransaminase & 12.0 & $\mathrm{IU} / \mathrm{L}$ & $\mathrm{pH}$ & 5.5 & \\
\hline Basophils & 0.8 & $\%$ & Total bilirubin & 0.4 & $\mathrm{mg} / \mathrm{dL}$ & Glucose & Negative & \\
\hline Monocytes & 7.3 & $\%$ & Gamma-glutamyl transferase & 20.0 & $\mathrm{IU} / \mathrm{L}$ & Protein & Negative & \\
\hline Hemoglobin & 12.3 & $\mathrm{~g} / \mathrm{dL}$ & Alkaline phosphatase & 341.0 & IU/L & Bilirubin & Negative & \\
\hline Hematocrit & 39.1 & $\%$ & Lactate dehydrogenase & 216.0 & $\mathrm{IU} / \mathrm{L}$ & Ketones & + & \\
\hline \multirow[t]{2}{*}{ Platelets } & 40.5 & $10^{4} / \mu \mathrm{L}$ & Urea nitrogen & 12.4 & $\mathrm{mg} / \mathrm{dL}$ & Hemoglobin & Negative & \\
\hline & & & Creatinine & 0.6 & $\mathrm{mg} / \mathrm{dL}$ & Nitrate & Negative & \\
\hline Coagulation test & & & Sodium & 134.0 & $\mathrm{mEq} / \mathrm{L}$ & Leukocyte esterase & + & \\
\hline Prothrombin time & 85.7 & $\%$ & Potassium & 4.3 & $\mathrm{mEq} / \mathrm{L}$ & Microscopy exam & & \\
\hline International normalized ratio & 1.1 & & Chloride & 93.0 & $\mathrm{mEq} / \mathrm{L}$ & Red blood cells & $1-4$ & /HPF \\
\hline D-Dimer & 1.5 & $\mu \mathrm{g} / \mathrm{mL}$ & Calcium & 9.3 & $\mathrm{mg} / \mathrm{dL}$ & White blood cells & $1-4$ & /HPF \\
\hline Fibrinogen & 452.0 & $\mathrm{mg} / \mathrm{dL}$ & Phosphate & 3.1 & $\mathrm{mg} / \mathrm{dL}$ & Epithelial cells & $1-4$ & /HPF \\
\hline \multirow[t]{13}{*}{ Fibrin degradation products } & 3.9 & $\mu \mathrm{g} / \mathrm{mL}$ & Creatine kinase & 92.0 & $\mathrm{IU} / \mathrm{L}$ & Casts & $1-4$ & /HPF \\
\hline & & & C-reactive protein & 1.6 & $\mathrm{mg} / \mathrm{dL}$ & Crystals & Negative & \\
\hline & & & Procalcitonin & 0.1 & $\mathrm{ng} / \mathrm{mL}$ & & & \\
\hline & & & Beta-D-glucan & 24.0 & $\mathrm{pg} / \mathrm{mL}$ & & & \\
\hline & & & Interferon-gamma release assays & Negative & & Cultures & & \\
\hline & & & Triglyceride & 91.0 & $\mathrm{mg} / \mathrm{dL}$ & Blood of aerobic & Negative & \\
\hline & & & Total cholesterol & 108.0 & $\mathrm{mg} / \mathrm{dL}$ & Blood of anaerobic & Negative & \\
\hline & & & LDL-cholesterol & 54.0 & $\mathrm{mg} / \mathrm{dL}$ & Urine & Negative & \\
\hline & & & HDL-cholesterol & 41.0 & $\mathrm{mg} / \mathrm{dL}$ & Removed implanted catheter & $*$ & \\
\hline & & & $\mathrm{HbA} 1 \mathrm{c}$ & 5.9 & $\%$ & Aneurysm pus & * & \\
\hline & & & HBs antigen & Negative & & & & \\
\hline & & & HCV antibody & Negative & & & & \\
\hline & & & HIV antigen/antibody & Negative & & & & \\
\hline
\end{tabular}

*Refer to Table 2

adventitia and infiltration of neutrophils, lymphocytes, and plasma cells in the media of the blood vessels (Fig. 2). On the basis of these findings, a diagnosis of catheterrelated thoracoabdominal infected aortic aneurysm was made.

On the day of admission, antibiotics therapy was considered for implanted catheter-related infection. The patient was administered a combination of vancomycin $1.0 \mathrm{~g}$ intravenously every 12 hours, piperacillin/tazobactam 4.5 g every 6 hours, and micafungin $150 \mathrm{mg}$ every 24 hours until all culture results were confirmed for a week at first. After determination of the drug susceptibilities of all strains, these antibiotics were found to be suitable and were continued. Although the thoracoabdominal aneurysm was resected and pus was drained, antibiotics were administered for 6 weeks in consideration of infection of the perivascular area from the celiac artery to the hepatic artery. His symptoms and laboratory test results improved after surgery and administration of antibiotics. The patient was discharged on day 45 , and no recurrence of infected aortic aneurysm was observed on subsequent follow-up CTs as an outpatient for 1 year.

\section{Discussion}

Although there are some reports of infections and pseudoaneurysms due to catheters [4], to the best of our knowledge, no study has reported infected thoracoabdominal aneurysms that are associated with implanted long-term arterial catheters for chemotherapy. This case was a rare but extreme scenario caused by a long-term indwelling aortic catheter compounded by rare organisms responsible for the infection.

The most prevalent microorganisms present in the arterial wall that are likely to cause infection of an aneurysm are Staphylococcus spp. and Salmonella spp. [5]. Other microorganisms include Streptococcus pneumoniae, Treponema pallidum, and Mycobacterium tuberculosis, as well as other bacterial, fungal, and anaerobic pathogens [6]. Infected aneurysms can occur in any artery but are most observed in the extremities, 

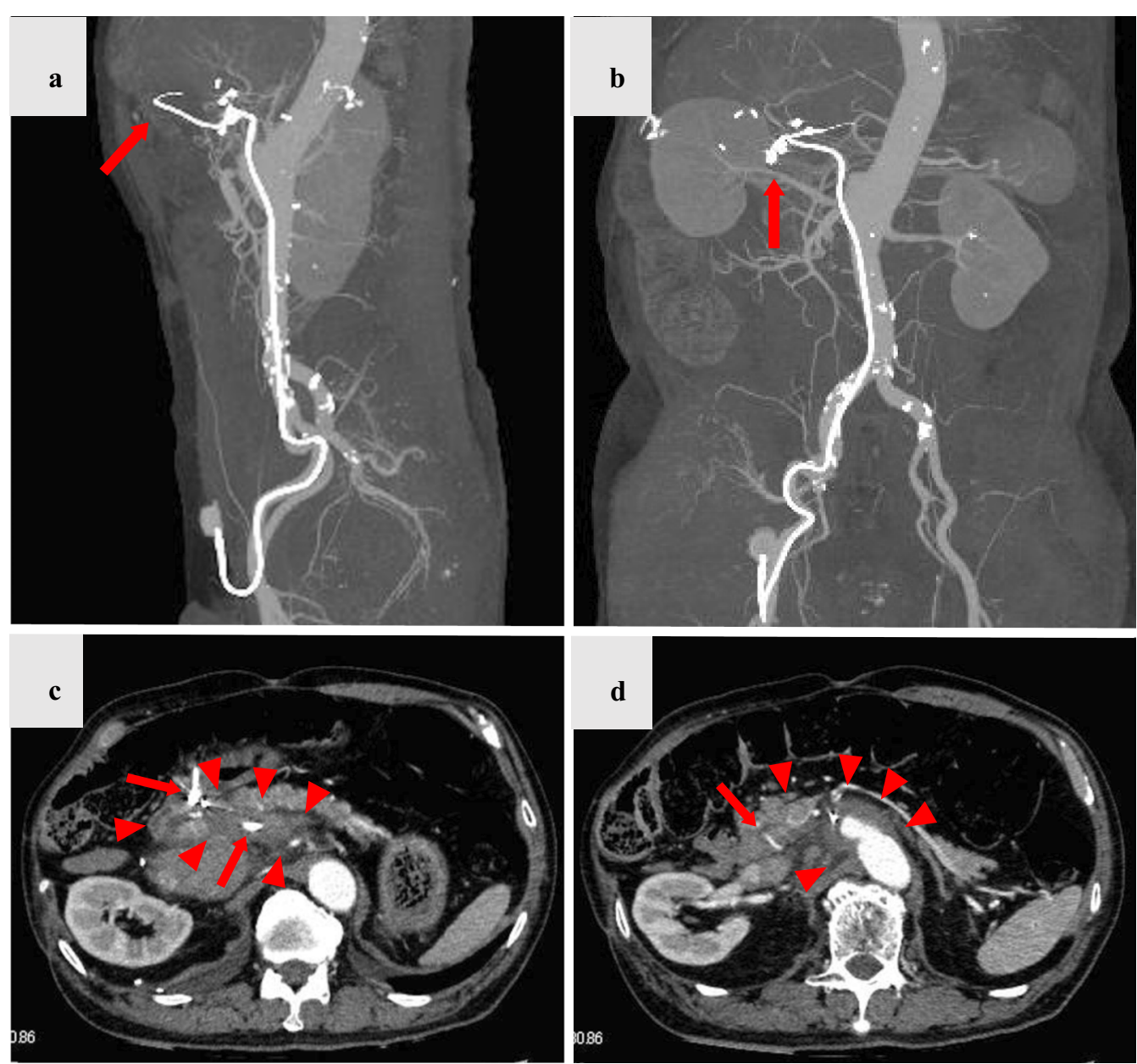

Fig. 1 Computed tomography of the chest and abdomen reveals the thoracoabdominal aortic aneurysm along with the implanted arterial catheter inserted from the left femoral artery to the hepatic artery ( $\mathbf{a}, \mathbf{b}$ arrow). An irregularly shaped aortic aneurysm was identified at the origin of the celiac artery, with partially expanded common hepatic artery with disproportionate fat stranding (c, $\mathbf{d}$ arrowhead) along the catheter (c, $\mathbf{d}$ arrow); no extravasation was observed using contrast enhancement (c, d)

splanchnic, and cerebral circulations, often at the points of vessel bifurcation [7]. In the present case, cultures of the aortic aneurysm wall tissue revealed the causative pathogens as E. coli, S. marcescens, E. corrodens, S. anginosus, $\alpha$-Streptococcus, and C. glabrata. There were several possible routes of infection related to implanted long-term arterial catheters, and the more likely ones were through the transhepatic artery, transhepatic portal vein, and transdermal infections, including bloodstream infections. Although cultures from the removed catheter and aneurysm pus revealed several pathogens, transient bacteremia associated with an implanted arterial catheter could have been controlled by autoimmunity. In addition, multiple pathogens may exist in the aortic arterial wall and may have caused the infected aortic aneurysm. Interestingly, it is considered that the celiac artery had a damaged arterial wall during insertion of the catheter, which eventually developed into an aneurysm, with inflammation and microorganisms spreading to the thoracoabdominal aorta. This was an extremely rare occurrence, and an infectious aortic aneurysm was suspected from the distortion of the aneurysm shape.

Several reports have investigated the risk factors for an infected aneurysm, and these include arterial injury, trauma, antecedent infection, endocarditis, preexisting aneurysm, impaired immunity, and advanced age $[8,9]$. The patient's past medical history and clinical examination did not indicate the presence of these risk factors. Either a venous or arterial indwelling catheter has an obviously high risk of catheter-related infection. In general, the diagnosis of an infected aneurysm is based upon imaging the aneurysm, and infection is confirmed 


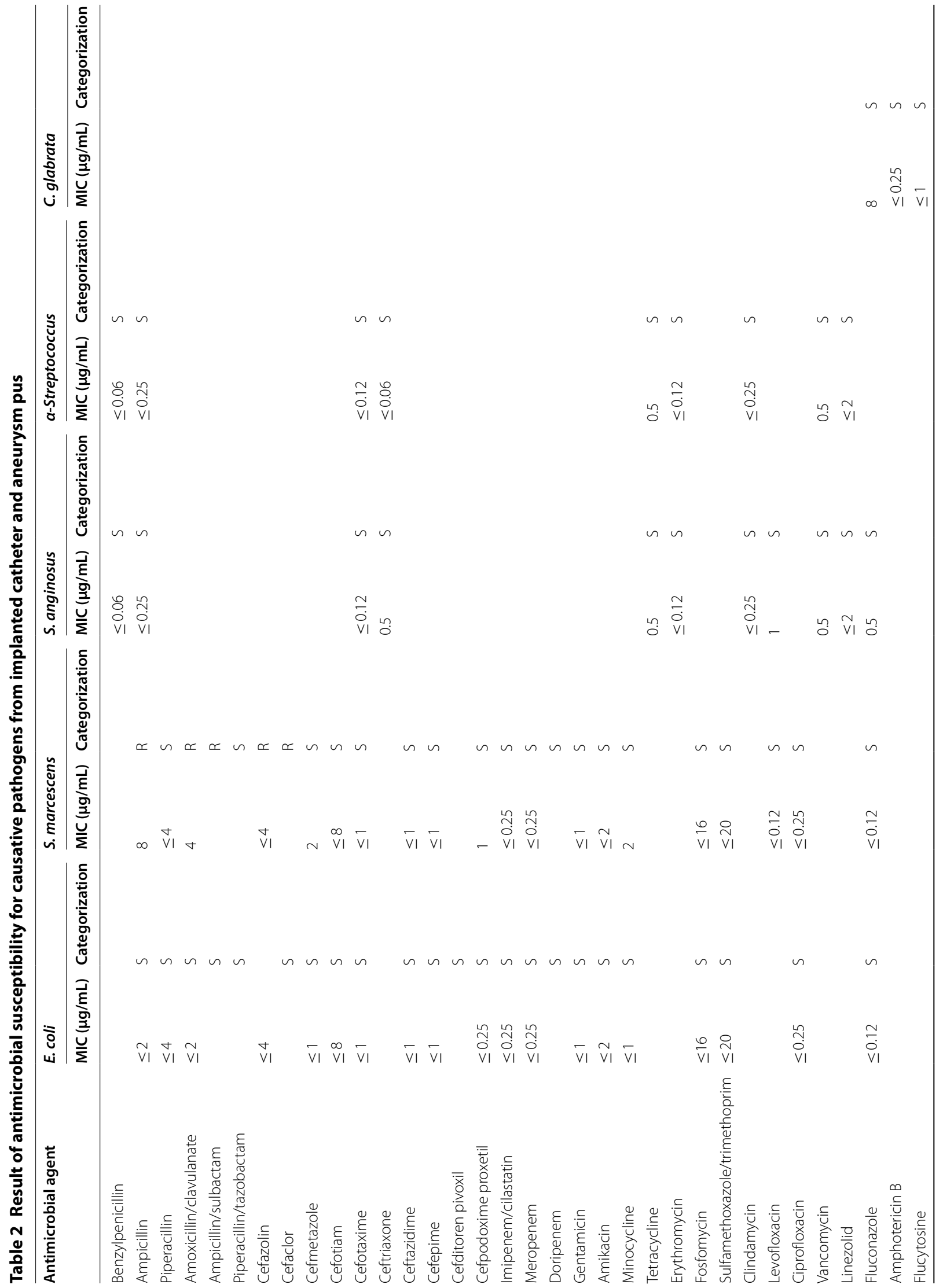




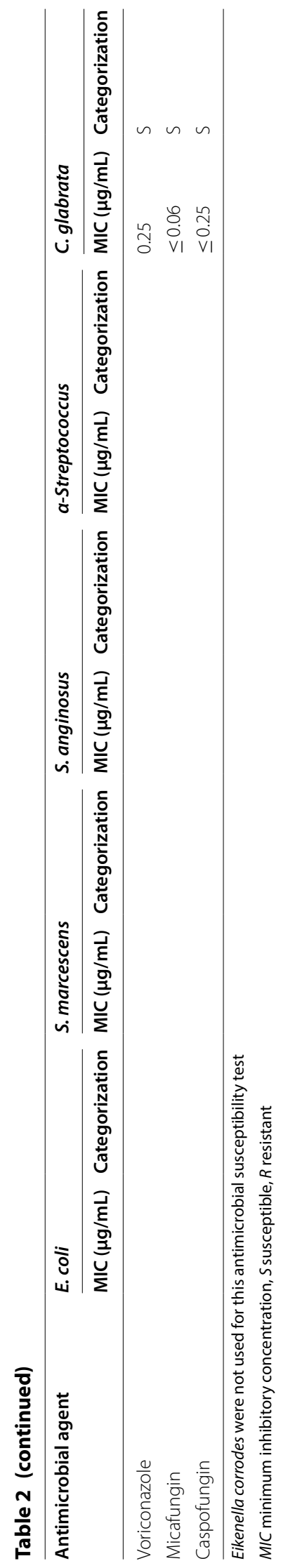




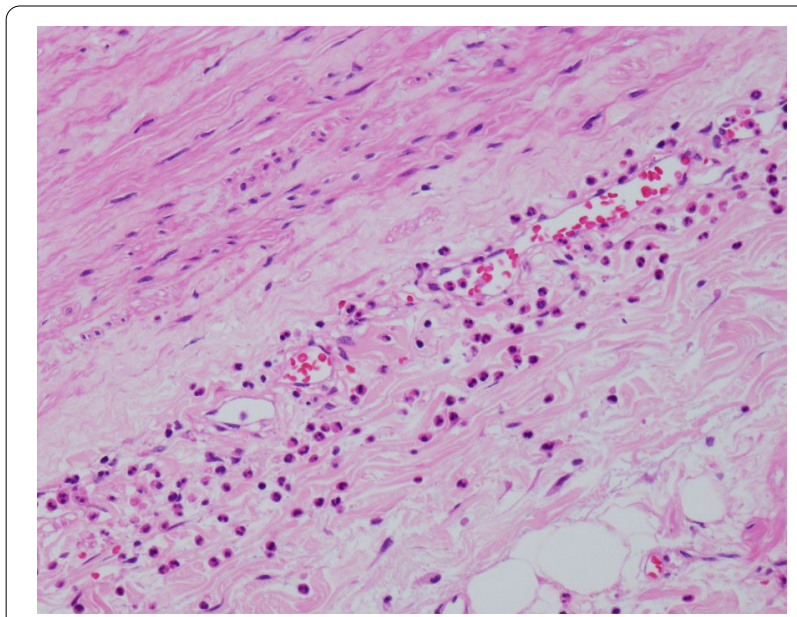

Fig. 2 Histopathology examination of the aortic aneurysm wall confirmed an infected aortic aneurysm based on infiltration of neutrophils mainly in the small blood vessels around the adventitia and infiltration of neutrophils, lymphocytes, and plasma cells in the media of the blood vessels (hematoxylin and eosin staining) by culturing an organism from the blood. CT angiography definitively diagnoses the aneurysm, specific features suggest infection, and CT also simultaneously evaluates the status of the circulation [10]. In this case, we considered that the thoracoabdominal aortic aneurysm was highly likely to be related to the implanted catheter on the basis of findings from CT imaging, the catheter and pus from aortic wall tissue cultures, and pathology examinations.

The standard treatment of most infected aneurysms is antibiotic therapy combined with surgical debridement with or without revascularization [11]. The initial choice of antibiotic therapy should be guided by the most likely infecting organism on the basis of the clinical circumstances. Antibiotics should be tailored to culture and susceptibility results when they become available. If surgical drainage is performed, this time period commences from the day of surgery. However, there are no data to support a specific duration of antibiotic therapy. In this case, we administrated a combination of antibiotics for 6 weeks on the basis of physical examination, laboratory findings, and follow-up CT findings.

In this era, because the number of patients has been increasing with catheter-based examination and treatment options, the number of patients with indwelling catheter infections is also expected to increase. Immediate confirmatory diagnosis and appropriate treatment are essential. As a matter of course, it is important to remove the catheter as soon as possible at the end of procedure to avoid this critical illness.

\section{Conclusion}

This case was a rare but extreme scenario caused by a long-term indwelling catheter compounded by rare organisms responsible for the infection. Clinicians should be aware that long-term implanted arterial catheters can cause not only catheter-related bloodstream infections but may also be a risk factor for infected aortic aneurysms in rare cases.

\section{Acknowledgements \\ Not applicable.}

\section{Authors' contributions}

KT wrote the initial and contributed to data collection and interpretation and critically reviewed the manuscript draft of the manuscript. All authors read and approved the final version of the manuscript and agree to be accountable for all aspects of the work in ensuring that questions related to the accuracy or integrity of any part of the work are appropriately investigated and resolved.

\section{Funding}

None.

\section{Availability of data and materials}

Not applicable.

\section{Ethics approval and consent to participate}

This study was conducted in the fundamental principles of the Declaration of Helsinki.

\section{Consent for publication}

Written informed consent was obtained from the patient for publication of this case report and any accompanying images. A copy of the written consent is available for review by the Editor-in-Chief of this journal.

\section{Competing interests}

The authors declare that they have no competing interests.

\section{Author details}

${ }^{1}$ Division of Critical Care Medicine, Nara Prefecture General Medical Center, Shichijyonishi 2-897-5, Nara City, Nara, Japan. ${ }^{2}$ Department of Critical Care Medicine, JA Hiroshima General Hospital, Hiroshima, Japan. ${ }^{3}$ Department of Cardiovascular Surgery, Nara Prefecture General Medical Center, Nara City, Nara, Japan.

Received: 23 January 2020 Accepted: 4 January 2021

Published online: 21 February 2021

\section{References}

1. Kim YW. Infected aneurysm: current management. Ann Vasc Dis. 2010;3:7-15.

2. Bennett DE. Primary mycotic aneurysms of the aorta: report of case and review of the literature. Arch Surg. 1967:94:758-65.

3. Mundth ED, Darling RC, Alvarado RH, Buckley MJ, Linton RR. Surgical management of mycotic aneurysms and the complications of infection in vascular reconstructive surgery. Am J Surg. 1968;117:460-70.

4. Katsuragawa F, Nagahama K, Naito S, et al. Ruptured infected aneurysm of the thoracic aorta associated with tunneled dialysis catheter-related 
methicillin-resistant Staphylococcus aureus bacteremia in a hemodialysis patient. CEN Case Rep. 2018;7:325-9.

5. Johnson JR, Ledgerwood AM, Lucas CE. Mycotic aneurysm. New concepts in therapy. Arch Surg. 1983;1 18:577-82.

6. Hsu RB, Chen RJ, Wang SS, Chu SH. Infected aortic aneurysms: clinical outcome and risk factor analysis. J Vasc Surg. 2004;40:30-5.

7. Baddour LM, Wilson WR, Bayer AS, et al. Infective endocarditis in adults: diagnosis, antimicrobial therapy, and management of complications: a scientific statement for healthcare professionals from the American Heart Association. Circulation. 2015;132:1435-86.

8. Chan YC, Ting AC, Law S, Cheng SW. Secondary infection of a preexisting thoracic aortic aneurysm by iatrogenic oesophageal perforation with aorta-oesophageal fistula formation. Eur J Cardiothorac Surg. 2009:35:365-7.
9. Oderich GS, Panneton JM, Bower TC, et al. Infected aortic aneurysms: aggressive presentation, complicated early outcome, but durable results. J Vasc Surg. 2001;34:900-8.

10. Wilson WR, Bower TC, Creager MA, et al. Vascular graft infections, mycotic aneurysms, and endovascular infections: a scientific statement from the American Heart Association. Circulation. 2016;134:e412-60.

11. Reddy DJ, Shepard AD, Evans JR, Wright DJ, Smith RF, Ernst CB. Management of infected aortoiliac aneurysms. Arch Surg. 1991;126:873-8.

\section{Publisher's Note}

Springer Nature remains neutral with regard to jurisdictional claims in published maps and institutional affiliations.
Ready to submit your research? Choose BMC and benefit from:

- fast, convenient online submission

- thorough peer review by experienced researchers in your field

- rapid publication on acceptance

- support for research data, including large and complex data types

- gold Open Access which fosters wider collaboration and increased citations

- maximum visibility for your research: over $100 \mathrm{M}$ website views per year

At BMC, research is always in progress.

Learn more biomedcentral.com/submissions 